\title{
PREDICTING THE SPATIAL DYNAMICS OF WOLBACHIA INFECTIONS IN AEDES AEGYPTI ARBOVIRUS VECTOR POPULATIONS IN HETEROGENEOUS LANDSCAPES
}

Penelope A. Hancock ${ }^{\mathrm{a}^{*}}$, Scott A. Ritchieb, Constantianus J. M. Koenraadtc, Thomas W. Scottd ${ }^{\mathrm{d}}$ Ary A. Hoffmann ${ }^{\mathrm{e}}$, H. Charles J. Godfraya

a. *Corresponding author. Department of Zoology, University of Oxford, Oxford, OX1 3PS, UK. Email: hancock.penelope@gmail.com

b. Australian Institute of Tropical Health and Medicine, James Cook University, 4870, Australia.

c. Laboratory of Entomology, University of Wageningen, 6700AA, The Netherlands.

d. Department of Entomology and Nematology, University of California, Davis, CA 95616-5270, USA.

e. Bio 21 Molecular Science and Biotechnology Institute, University of Melbourne, 3010, Australia.

\section{ABSTRACT}

1. A promising strategy for reducing the transmission of dengue and other arboviral human diseases by Aedes aegypti mosquito vector populations involves field introductions of the endosymbiotic bacteria Wolbachia. Wolbachia infections inhibit viral transmission by the mosquito, and can spread between mosquito hosts to reach high frequencies in the vector population. Wolbachia spreads by maternal transmission, and spread dynamics can be variable and highly dependent on natural mosquito population dynamics, population structure and fitness components.

2. We develop a mathematical model of an Ae. aegypti metapopulation that incorporates empirically validated relationships describing densitydependent mosquito fitness components. We assume that density dependence relationships differ across subpopulations, and construct heterogeneous landscapes for which model-predicted patterns of variation in mosquito abundance and demography approximate those observed in field populations. We then simulate Wolbachia release strategies similar to that used in field trials.

3. We show that our model can produce rates of spatial spread of Wolbachia similar to those observed following field releases.

4. We then investigate how different types of spatio-temporal variation in mosquito habitat, as well as different fitness costs incurred by Wolbachia on the mosquito host, influence predicted spread rates. We find that fitness costs reduce spread rates more strongly when the habitat landscape varies temporally due to stochastic and seasonal processes.

5. Our empirically based modelling approach represents effects of environmental heterogeneity on the spatial spread of Wolbachia. The models can assist in interpreting observed spread patterns following field releases and in designing suitable release strategies for targeting spatially heterogeneous vector populations. 


\section{KEYWORDS}

arbovirus, dengue, gene drive, spatial spread, $w \mathrm{AlbB}, w \mathrm{Mel}, w \mathrm{MelPop}$, Zika

\section{INTRODUCTION}

Aedes aegypti mosquitoes transmit several arboviruses, including dengue which infects an estimated 390 million people per year (Bhatt et al. 2013), as well as Zika and chikungunya which have expanded geographically on a global scale in recent years (Nunes et al. 2015; Faria et al. 2017). Vaccines against these viruses are not yet widely available (Saez-Llorens et al. 2017) and disease control strategies rely on interventions that target the mosquito vector. A promising new control strategy involves the field releases of the endosymbiotic bacteria Wolbachia into Ae. aegypti populations. Wolbachia infections in Ae. aegypti inhibit virus development and their transmission by the vector (Moreira et al. 2009; Walker et al. 2011). Wolbachia is maternally transmitted in the mosquito host, and utilizes a mechanism known as cytoplasmic incompatibility (CI), which drives the invasion of the bacteria throughout the population. CI works by preventing the development of offspring from matings between Wolbachiainfected males and uninfected females (Caspari \& Watson 1959), conferring a strong relative fitness advantage to infected females that are able to mate with both infected and uninfected males. Field releases conducted in northeast Australia in 2011 achieved the first successful invasion and stable establishment of a Wolbachia strain (wMel) in wild Ae aegypti populations (Hoffmann et al. 2011). Current field trials are evaluating the public health impact, as measured by reductions in human disease incidence, of Wolbachia infected Ae. aegypti (0"Neill et al. 2018).

Given the growing need and potential for applying Wolbachia to mosquito vector control and disease prevention, it is necessary to understand the dynamics of Wolbachia spread in field Ae. aegypti populations. The spatial spread of Wolbachia is expected to depend on the natural heterogeneity in mosquito abundance and demography that arises from changing ecological and environmental conditions (Hancock et al. 2016a; Hancock et al. 2016b). Theoretical studies demonstrate that spatial heterogeneity in host abundance is critical to Wolbachia spread, slowing and sometimes stopping the invasion (Barton 1979; Hancock \& Godfray 2012). Demographic variation amongst individual hosts, and how this is spatially structured, also has important consequences for spread because the rate of maternal transmission of Wolbachia depends directly on its hosts' fitness (Hancock et al. 2016b).

In mosquito populations, important demographic traits such as female fecundity and larval development rates can vary dramatically depending on the levels of density-dependent competition for limited food resources that are experienced during larval development (Barrera et al. 2006; Muriu et al. 2013; Hancock et al. 2016a). Parameters describing female fecundity and larval development rates are fundamental to models of Wolbachia dynamics in mosquito populations, because the bacteria is only transmitted maternally (Turelli 1994). Incorporation of density-dependent demographic variation in these parameters into models of the spatial dynamics of Wolbachia has, however, been hindered by the lack of an empirical understanding of the form of density dependence (Legros et al. 2009). 
Models of the spatial spread of Wolbachia in mosquito populations developed thus far have typically either assumed constant demographic rates across space and time (Turelli \& Hoffmann 1991; Schmidt et al. 2017; Turelli \& Barton 2017), or restricted the effects of density-dependence to impacts on larval survival (Hancock \& Godfray 2012).

Here we develop a spatially explicit model of Wolbachia-Ae. aegypti dynamics that aims to incorporate realistic patterns of demographic variation in the mosquito population. Ae. aegypti is a highly domestic species that is found in or near areas with human habitation and has low rates of dispersal (Harrington et al. 2005), which makes population size estimation easier than for other mosquito species (Ritchie et al. 2013a). Studies from multiple geographic regions show characteristic strong spatial overdispersion in population size (Getis et al. 2003; Focks \& Alexander 2006; Koenraadt et al. 2008; Jeffery et al. 2009; Aldstadt et al. 2011; LaCon et al. 2014), which may arise from the reliance of Ae. aegypti larvae on developing in water filled artificial containers close to human dwellings (Southwood et al. 1972; Aldstadt et al. 2011; Williams et al. 2013). Many of these containers produce low numbers of pupae (and adults), which is thought to be due limited larval food resources (Arrivillaga \& Barrera 2004; Focks \& Alexander 2006). Adult body size in field populations is typically smaller than those produced from larvae that are fed ad libitum in the laboratory (Nasci 1986; Barrera et al. 2006), with more crowded field containers typically producing smaller adults (Schneider et al. 2004). This indicates that larval development in field Ae. aegypti populations is affected by food limitation arising from densitydependent larval competition, which adversely affects juvenile and adult fitness components (Hancock et al. 2016a). Thus, density dependent effects on mosquito production and fitness could be an impediment for Wolbachia to establish and spread, especially as releases of additional mosquitoes will lead to increased crowding in containers.

Wolbachia spread may also be impacted by spatio-temporal variation in mosquito habitat (Schmidt et al. 2017). Field studies have shown that over time it is not always the same houses that are responsible for the production of high numbers of Ae. aegypti (Jeffery et al. 2009; LaCon et al. 2014), suggesting that the quality of mosquito habitat within a given house may be temporally variable. In some environments, including northeast Australia, Ae. aegypti abundance is seasonal and higher in the wet season (November-April) than in the dry season (May-October) (Ritchie et al. 2013b). In some other environments, however, Ae. aegypti abundance does not show seasonal variation (Scott et al. 2000b; Koenraadt et al. 2008).

We develop a metapopulation model that describes the spatially heterogeneous demography of Ae. aegypti using empirically validated relationships that link density-dependent demographic traits to mosquito abundance. Incorporating an empirically realistic form of density-dependence significantly reduces parameter uncertainty compared to other models of Ae. aegypti population dynamics. We show that our model can produce spatially aggregated patterns of demographic variation similar to those observed in field populations, and that it predicts rates of spatial spread of Wolbachia close to those observed following field releases. 
We then explore the effects on the rate of spread of different types of spatiotemporal variation in the mosquito habitat landscape, including stochastic and seasonal variation, and of different fitness costs incurred by Wolbachia on the mosquito host.

\section{METHODS}

We develop an age-structured model of an Ae. aegypti metapopulation that assumes that variation amongst individuals in two demographic traits, larval development times and per-capita female fecundity, is influenced by varying levels of larval density-dependent competition for limited food resources (Hancock et al. 2016b). The level of density-dependent competition within each of the subpopulations that make up the metapopulation is assumed to depend on the local larval density as well as the quantity of larval food resources available to the subpopulation (Fig 1A). We conceptualize each subpopulation of the metapopulation as a "house" and define the larval habitat quality in each house as high quality, low quality or empty (no larval habitat present). High quality habitats contain a greater larval food resource quantity than low quality habitats.

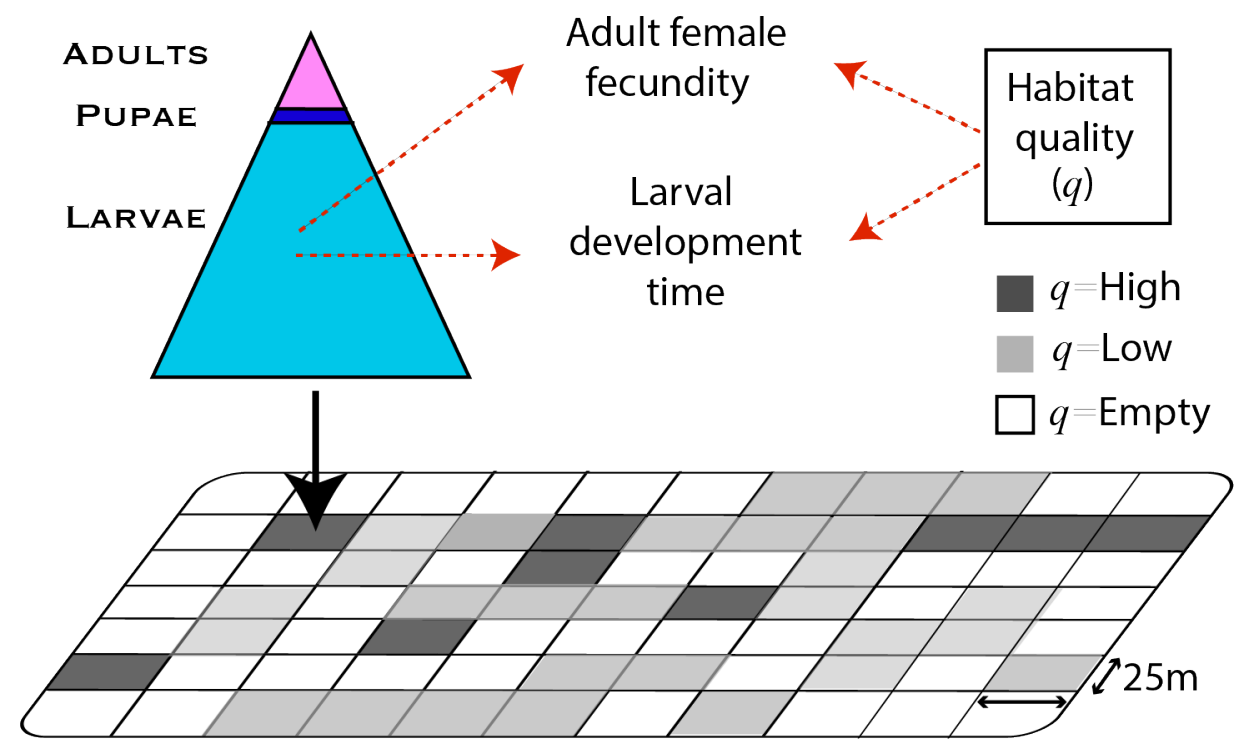

Figure 1. Diagram of the metapopulation model showing interactions between mosquito demographic traits, larval density and habitat quality across subpopulations.

In order to estimate the functional form of density-dependent demographic variation, we utilize a recently developed methodology that is based on frequent observations of the abundance of the juvenile life stages of Ae. aegypti in two experimental field-caged populations over a period of approximately six months (see below and Hancock et al. (2016b)). One field-caged population received a higher quantify of larval food resources per week and the other a lower quantity (see below). Mathematical functions describing the density-dependent covariation in per-capita female fecundity and larval development times occurring in each population are then parameterized from the observed variation in juvenile abundance (Hancock et al. 2016b). We use these two sets of relationships to represent the form of density dependence occurring in the high and low quality habitats in our metapopulation model. The other model parameters, including those describing density-independent mosquito 
demographic traits and dispersal, are assumed to be constant and are estimated using published data.

We describe below the details of our methodology for estimating the densitydependent and density-independent model parameters. Parameters values are provided in Tables $1 \& 2$ and the mathematical formulation of the model is given in Section S4. We then present approaches for comparing patterns of demographic variation in our simulated metapopulation to those observed in field Ae. aegypti populations. Finally we describe our method for comparing predicted rates of spatial spread of $w$ Mel Wolbachia to those observed in the field.

\section{Estimating the form of density-dependent demographic variation}

We estimated the forms of density-dependent variation in mosquito demographic traits occurring in two independent experimental populations of Ae. aegypti. Each population was initiated with a cohort of 100 first instar larvae that were the progeny of wild caught Ae. aegypti collected in Cairns, northeast Australia. The populations were housed in field-cages designed to simulate the natural habitat of Ae. aegypti and the climatic conditions of northeast Australia (Darbro et al. 2012) and maintained as described in Hancock et al. (2016a; 2016b) (see Section S1 of the Supplementary Material). Each population received a different larval food regime. One population, which we refer to as the HF (high food) Population, received a fixed quantity of food $(0.32 \mathrm{~g}$ ground lucerne) three times per week, and the other, referred to as the LF (low food) Population, received this same food quantity only once per week.

We monitored the abundance of larvae and pupae over approximately six months (194 days for the HF Population and 187 days for the LF Population). All newly eclosed pupae were counted daily and all larvae (categorized as first, second, third and fourth instar) were counted three times per week. We used Bayesian Markov Chain Monte Carlo (MCMC) methods, described in Hancock et al. (2016b), to estimate the parameters of mathematical functions describing the form of larval density-dependent variation in larval development times and percapita female fecundity for each population. These methods calculate the likelihood of our observed abundances of the juvenile mosquito life stages over time. Details of this methodology are provided in Section S2. For both populations, the fitted models accurately describe the major features of the temporal variation in juvenile mosquito abundances in the field-caged populations (Fig S1). For the HF Population, these model fits have been reported previously (Hancock et al. 2016b), while for the LF Population our results are reported here for the first time. We note that incorporating density-dependent variation in additional demographic traits, such as the daily larval mortality rate, may have produced a closer fit to the field cage observations.

\section{Estimating density-independent demographic traits}

Values of parameters describing density-independent demographic rates (assumed constant) were estimated from published studies (Table 1). We estimated the daily probability, $p_{d}$ that adults move to an adjacent house (within the Moore neighbourhood), using published data from mark-release-recapture 
(MRR) experiments performed in Thailand (lines 1 and 2 of Table 2 of Harrington et al. (2005)) that record numbers of recaptured Ae. aegypti mosquitoes at different distances from the release sites. We simulated the MRR protocol described in Harrington et al. assuming a constant daily rate of adult mosquito mortality $\mu_{A}$ (Table 1 ). We calculated the value of $p_{d}$ that provided the minimum least squares fit to the observed recapture rates. We obtained $p_{d}=$ 0.56 , which is higher than the estimated daily movement probability of 0.3 obtained by Magori et al. (2009), who also simulated the Harrington et al. MRR data but defined the set of neighbouring houses as a von Neumann neighbourhood. We assumed that larvae experience a constant daily rate of mortality $\mu_{L}$ similar to the value estimated for our field-cage experiments (Hancock et al. 2016b), and that pupae experience the same daily mortality rate as adults. Values of the development time lags $T_{G}, T_{H}$ and $T_{P}$ were estimated from our field-cage experiments (Hancock et al. 2016b).

Table 1. Values of model parameters describing mosquito demographic traits and dispersal.

\begin{tabular}{|c|c|c|c|c|}
\hline Symbol & Definition & \multicolumn{2}{|c|}{ Value } & Source \\
\hline$T_{P}$ & Duration of the pupal stage & \multicolumn{2}{|c|}{2 days } & $\begin{array}{c}\text { (Hancock et al. } \\
\text { 2016a) }\end{array}$ \\
\hline$T_{G}$ & $\begin{array}{l}\text { Minimum time for females to } \\
\text { become gravid following } \\
\text { emergence }\end{array}$ & \multicolumn{2}{|c|}{4 days } & $\begin{array}{c}\text { (Hancock et al. } \\
\text { 2016a) }\end{array}$ \\
\hline$T_{H}$ & $\begin{array}{l}\text { Time between oviposition and } \\
\text { hatching of eggs }\end{array}$ & \multicolumn{2}{|c|}{6 days } & $\begin{array}{c}\text { (Hancock et al. } \\
\text { 2016a) }\end{array}$ \\
\hline$\omega$ & $\begin{array}{l}\text { The proportion of uninfected } \\
\text { offspring produced by } \\
\text { Wolbachia-infected females }\end{array}$ & \multicolumn{2}{|c|}{0.01} & $\begin{array}{c}\text { (Walker et al. } \\
\text { 2011) }\end{array}$ \\
\hline$S_{h}$ & $\begin{array}{l}\text { The proportion of unviable } \\
\text { offspring from an incompatible } \\
\text { mating }\end{array}$ & \multicolumn{2}{|c|}{0.99} & $\begin{array}{c}\text { (Walker et al. } \\
\text { 2011) }\end{array}$ \\
\hline$S$ & $\begin{array}{l}\text { The reduction in average adult } \\
\text { lifespan caused by Wolbachia } \\
\text { carriage }\end{array}$ & \multicolumn{2}{|c|}{ varies } & \\
\hline \multirow[b]{2}{*}{$\alpha, \beta, \gamma$} & \multirow[b]{2}{*}{$\begin{array}{l}\text { Parameters of functions } \\
\text { describing mean larval } \\
\text { development time }\end{array}$} & $\begin{array}{l}\text { Low quality } \\
\text { habitats }\end{array}$ & $\begin{array}{l}\text { High quality } \\
\text { habitats }\end{array}$ & \\
\hline & & $\begin{array}{c}19.6,2.74 \\
0.43\end{array}$ & $\begin{array}{c}1.2,0.154 \\
0.68\end{array}$ & This study \\
\hline$v, \eta, \psi$ & $\begin{array}{l}\text { Parameters of functions } \\
\text { describing the standard deviation } \\
\text { of larval development time }\end{array}$ & $\begin{array}{c}-0.97,1.0 \\
0.32\end{array}$ & $\begin{array}{c}12,0.24 \\
0.44\end{array}$ & This study \\
\hline$a, b$ & $\begin{array}{l}\text { Parameters of functions } \\
\text { describing per-capita female } \\
\text { fecundity }\end{array}$ & $15.4,-2.03$ & $28.5,-3.25$ & This study \\
\hline
\end{tabular}




\begin{tabular}{|c|c|c|c|c|}
\hline $\begin{array}{l}\lambda_{\min }, \mu_{\max } \\
\sigma_{\max }\end{array}$ & $\begin{array}{l}\text { Lower limit on per-capita female } \\
\text { fecundity, and upper limits on } \\
\text { larval development time means } \\
\text { and standard deviations }\end{array}$ & \multicolumn{2}{|c|}{$0.5,60$ days, 40 days } & This study \\
\hline$\mu_{L}^{c}$ & Daily larval mortality & \multicolumn{2}{|c|}{0.05} & $\begin{array}{l}\text { (Hancock et al. } \\
\text { 2016a) }\end{array}$ \\
\hline \multirow[t]{2}{*}{$\mu_{A}^{c}$} & Daily pupal and adult mortality & Field-cage & $\begin{array}{l}\text { Field release } \\
\text { simulations }\end{array}$ & \multirow{2}{*}{$\begin{array}{l}\text { (Muir \& Kay 1998; } \\
\text { Walker et al. } \\
\text { 2011) }\end{array}$} \\
\hline & & 0.03 & 0.1 & \\
\hline$p_{d}$ & $\begin{array}{l}\text { Daily probability that adults } \\
\text { move to adjacent house }\end{array}$ & \multicolumn{2}{|c|}{0.56} & $\begin{array}{l}\text { (Harrington et al. } \\
\text { 2005) }\end{array}$ \\
\hline
\end{tabular}

Table 2. Values of model parameters describing spatial structure and the sizes of mosquito releases.

\begin{tabular}{|c|c|c|}
\hline Symbol & Definition & Value \\
\hline$x_{h}$ & $\begin{array}{l}\text { Side length of square grid units } \\
\text { (houses) }\end{array}$ & $25 \mathrm{~m}$ \\
\hline$X_{A}$ & $\begin{array}{l}\text { Side length of square spatial region } \\
\text { containing the target mosquito } \\
\text { vector population }\end{array}$ & $2.5 \mathrm{~km}$ \\
\hline$h_{H}, h_{L}, h_{E}$ & $\begin{array}{l}\text { Proportions of houses with high, } \\
\text { low and empty habitat quality }\end{array}$ & $0.1,0.45,0.45$ \\
\hline$n_{r}$ & Number of releases & 15 \\
\hline$t_{r}$ & Duration of release period & 15 weeks \\
\hline$S_{r}$ & $\begin{array}{l}\text { Number of mosquitoes released } \\
\text { per release house per release* }\end{array}$ & $5^{*}$ \\
\hline$X_{r}$ & Size of the release zone* & $725 \mathrm{~m}^{2}$ \\
\hline$f_{r}$ & $\begin{array}{l}\text { Wolbachia frequency in the release } \\
\text { zone at the time of the final } \\
\text { release }^{*}\end{array}$ & 0.85 \\
\hline
\end{tabular}

*Used for model simulations where the fitness cost $s=0$ and the habitat quality landscape was static.

\section{Estimating spatial variation in habitat quality}

We denote the proportion of houses in the metapopulation with high habitat quality, low habitat quality and empty as $h_{H}, h_{L}$, and $h_{E}$. We assume that the habitat quality type across houses is spatially uncorrelated (Getis et al. 2003) and is described by a multinomial distribution $\operatorname{Mult}\left(h_{H}, h_{L}, h_{E}\right)$. We then compare the abundance of pupae across subpopulations simulated by our model with observations from field surveys conducted by Koenraadt et al. (2008). They recorded the number of pupae found across a set of residential properties distributed over two villages in Kamphaeng Phet province, Thailand, in four surveys conducted over 2 month periods at the end of the dry season (March and April, which is the beginning of the dengue transmission season) and at the end of the wet season (September and October, which is the end of the dengue transmission season) in 2004 and 2005. They made a total of 2123 observations 
across 604 houses. For each house, pupae in all water-holding containers were counted. Pupal abundances were determined for all potential larval development sites. The observed distribution of pupal numbers across houses across both surveys shows a spatially aggregated pattern that is typical for Ae. aegypti field populations (Getis et al. 2003; Focks \& Alexander 2006; Jeffery et al. 2009; LaCon et al. 2014).

We ran one-year simulations of our metapopulation model across which the only parameters that varied were the proportions of houses with high quality, low quality and empty habitats $\left(h_{H}, h_{L}\right.$, and $\left.h_{E}\right)$. We calculated the parameters of the multinomial habitat quality distribution for which the simulated distribution of pupal numbers per house best matched that observed (Table 2). Our approach assumed that the habitat quality of each house does not vary over time (see the Discussion). Further details of this methodology are provided in Section S3.

\section{Comparing the predicted intensity of density dependence with observations from field populations}

We assessed how the intensity of larval density-dependent competition occurring in our modelled metapopulation compared to observations from field populations using observed wing lengths of adult females produced from fieldcollected Ae. aegypti pupae. Food limitation during larval development greatly affects overall adult body size, which can be estimated using wing length (Briegel 1990; Barrera et al. 2006), so field populations that show smaller adult wing lengths are likely to have experienced more intense larval resource competition (Barrera et al. 2006). Temperature conditions can also affect Ae. aegypti body size (Scott et al. 2000a; Mohammed \& Chadee 2011), although in our field-cage populations changes in larval water temperature had less influence on demographic traits than variation in larval density (Hancock et al. 2016a) (see the Discussion). We used published observations of the average wing length per household of 2316 female Ae. aegypti collected as pupae from 2931 houses in Iquitos, Peru (Schneider et al. 2004). The wing lengths of the sampled mosquitoes are highly variable, and median wing length is low relative to that occurring when adult females experience plentiful food conditions during larval development (Fig. 1 of (Schneider et al. 2004)). Other observed wing lengths distributions obtained from field-sampled Ae. aegypti also show similar characteristics (Nasci 1986; Barrera et al. 2006).

In order to compare these wing length observations to the output of our model, we used the relationship between wing length and female fecundity obtained by Briegel (1990). Briegel (1990) recorded adult female wing length, $w$, and the number of eggs oviposited per gonotrophic cycle, $\tilde{\lambda}_{G}$, for 206 Ae. Aegypti females reared in a laboratory under varying larval density conditions and blood-fed on human hosts (Table 3 of Briegel 1990))). He then fitted a linear relationship to these observations:

$\tilde{\lambda}_{G}=2.505 w^{3}-8.616$

We applied eqn (1) to estimate the distribution of the average $\tilde{\lambda}_{G}$ per house, $\lambda_{G, h}$, from the observed distribution of average female wing lengths per house. This 
procedure assumes that individual wing lengths can be approximated by the house average. We then calculated the distribution of the weekly average percapita female fecundity for each house, $\lambda_{h}$, using observations from our fieldcage experiments (see Methods). This procedure makes two further assumptions: (i) $\lambda_{G, h}$ and $\lambda_{h}$ are assumed to be directly proportional; and (ii) the adult female wing lengths in our field-caged populations under conditions of plentiful larval food are equal to the highest value observed by Briegel (1990). Having obtained a distribution of per-capita female fecundity across individuals derived from the field wing length data, we compared it with the distribution of per-capita female fecundity values across all subpopulations simulated by our metapopulation model.

\section{Comparing the predicted rate of spatial spread of wMel Wolbachia with observations from field populations}

We compared the rates of spatial spread of Wolbachia predicted by our model to those observed following the $w$ Mel field release trials conducted at two sites in the city of Cairns, northeast Australia, in 2013 (Schmidt et al. 2017). The two sites, Edge Hill and Parramatta Park, are residential areas close to the center of Cairns. The field trials involved releasing mosquitoes infected with $w$ Mel into a restricted area, or "release zone", with the aim of allowing the Wolbachia to spread into surrounding areas. Observed rates of spread of $w$ Mel beyond the release zone for both sites are reported in Schmidt et al. (2017) (see Methods). We used their Gaussian/logistic model fits to observations of $w$ Mel frequencies in pooled samples (Fig. S3 of Schmidt et al. (2017)) to obtain the estimated distance from the edge of the release zone at which the $w$ Mel frequency was 0.5 at multiple time points following the releases.

To represent their spatial release strategy, we assume in our model that mosquitoes infected with $w \mathrm{Mel}$ are liberated within a square-shaped release zone located at the center of a larger area of potential habitat containing 10,000 houses (also square-shaped; $2.5 \mathrm{~km}^{2}$ ). We set parameters specifying the release protocol to values matching those used in the field trials (these included the area of the release zone, the duration and frequency of releases, and the frequency of $w$ Mel inside the release zone at the time of the final release; Table 2). We assumed that a fixed number of infected mosquitoes are released within each house inside the release zone once per week. Because released mosquitoes were reared in the laboratory, we also assumed that the per-capita fecundity of the released females is high and equal to that observed when larval food resources are plentiful (Hancock et al. 2016b). Our results assume that houses have dimensions of $25 \mathrm{~m}^{2}$ (Table 2), which is approximately equal to average area of the housing blocks within the two release zones in Cairns, calculated by randomly sampling 100 houses within each release zone and obtaining the dimensions of each housing block using Google Maps.

Our model of the Wolbachia dynamics in each subpopulation of the metapopulation is based on that described in Hancock et al. (2016b), and we provide the mathematical details in Section S5. We assume high values of the strength of cytoplasmic incompatibility, $s_{h}$, and the rate of maternal 
transmission, $\omega$ (Table 1), consistent with observations of the Wolbachia strain $w$ Mel that has been used in recent field release trials (Hoffmann et al. 2011). We begin by assuming that Wolbachia infection does not affect the fitness of its mosquito host (Hancock et al. 2016b), and then explore the effects of varying fitness costs on spread dynamics.

\section{RESULTS}

\section{Modelling spatially varying demography in the mosquito population}

\section{Modelling spatial variation in mosquito abundance}

The fitted distribution of the numbers of pupae across houses is similar in both shape and range to that obtained from the field surveys (Fig. 2A). The predicted pupal numbers are highly aggregated across space due to the small proportion of houses with high quality habitat $\left(h_{H}=0.1\right)$. The fitted proportions of houses with low and empty habitat quality $\left(h_{L}\right.$ and $\left.h_{E}\right)$ were equal (Fig. 2A). These results demonstrate that the relationships describing density-dependent demography derived from our field-caged population experiments can be applied directly, without rescaling, to approximate patterns of variation in abundance that occur in field Ae. aegypti populations.

\section{Modelling the intensity of density-dependent competition}

The median of the field-derived per-capita female fecundity distribution is in the lower part of the per-capita fecundity range and is close to that predicted by our model (Fig. 2B). Thus both field observations and model predictions indicate that density-dependent competition is relatively intense. While both the distributions from the field and model show wide variation in per-capita female fecundity across subpopulations, the variance predicted by our model is larger. The larval development times predicted by our model also vary widely across the metapopulation, with the majority of subpopulations having protracted larval development (Fig. 2B).
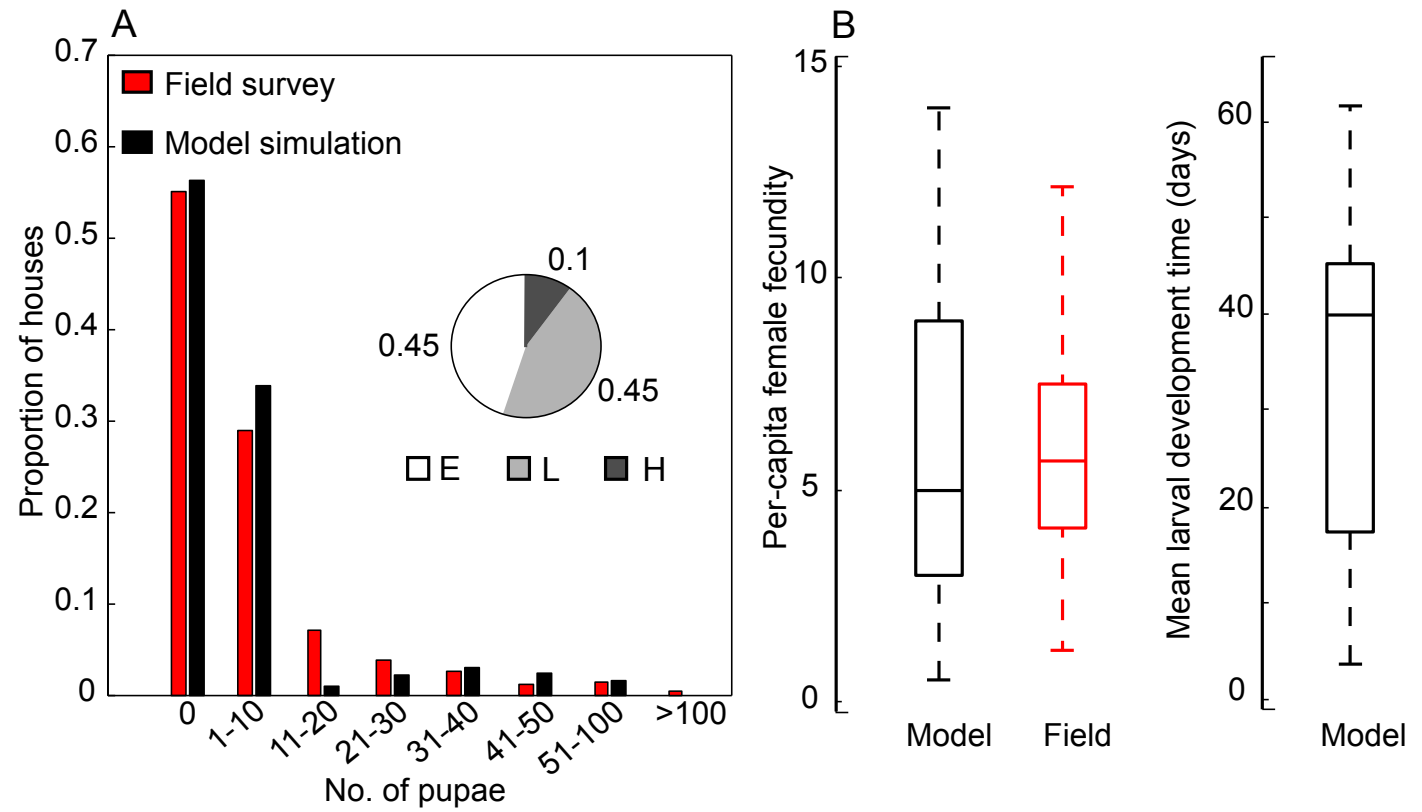

No. of pupae 
Figure 2. (A) The distribution of the number of pupae per house. Red bars show observations from the field surveys and black bars show the simulated distribution given by our metapopulation model. The pie chart shows the fitted proportions of houses with high $(\mathrm{H})$, low (L) and empty (E) habitat quality $\left(h_{H}, h_{L}\right.$, and $h_{E}$, respectively). (B) Boxplots showing the distribution of per-capita female fecundity across subpopulations predicted by our model (black, left), the per-capita fecundity distribution estimated from field-caught mosquitoes (red; see text), and the distribution of the mean development times of the larval cohorts predicted by our model (black, right).

\section{Predicting the speed of spatial spread of Wolbachia}

We simulated Wolbachia field releases for 50 landscapes where habitat quality in each house is randomly drawn from a multinomial distribution with parameters $h_{H}, h_{L}$, and $h_{E}$ (Table 1). The mean of the simulated rates of spread across all landscapes agrees well with the rates observed at Edge Hill and Parramatta Park (Fig. 3A). For each landscape the simulated spread rates beyond the release zone were spatially heterogeneous (Fig. 3A and see Animation S1) and the spatial spread pattern varied across the different landscapes.

\section{Effects of stochastic habitat variation}

We first extend our analysis to consider landscapes where habitat quality shows stochastic temporal variation. We defined a fixed daily probability $t_{q}$ that the habitat quality in each house is reassigned to a value chosen randomly from $\operatorname{Mult}\left(h_{H}, h_{L}, h_{E}\right)$. We then defined the expected habitat quality turnover period, $\bar{t}_{q}=1 / t_{q}$, as the expected time interval between a reassignment of habitat quality for each house, and considered cases where turnover is slow ( $\bar{t}_{q}=3$ months) and relatively fast ( $\bar{t}_{q}=1$ month). For each value of $\bar{t}_{q}$ we repeated the analysis described in the previous section to obtain predictions of the speed of spatial spread of Wolbachia by simulating the release protocol used in the field releases conducted in Edge Hill and Parramatta Park, Cairns (Table 2). We found that the predicted rate of spatial spread of Wolbachia for both values of $t_{q}$ was similar to that obtained when habitat quality was assumed not to vary temporally (Fig. 3BC).

\section{Effects of seasonal habitat variation}

We next extended our analysis to explore effects of seasonal variation in $A e$. aegypti abundance on Wolbachia spread. We simulated seasonal variation in Ae. aegypti abundance by periodically increasing the proportion of high quality habitat, $h_{H}$. This involved randomly selecting a set of houses (of size $n_{s}$ ) with either low or empty habitat quality, and reassigning the habitat quality in these houses to high, once every 365 days. This "wet season" habitat quality landscape remained fixed for 182 days, and then a set of high quality habitat houses, also of size $n_{s}$, was selected at random and the habitat quality in these houses was reassigned to either low, or empty, with a probability of 0.5 . We set the value of $n_{s}$ such that the proportion of houses with high quality habitat, $h_{H}$, increased to 0.175 at the start of the wet season (and returned to 0.1 at the start of the dry season). This produced a simulated pattern of seasonal variation in adult 
bioRxiv preprint doi: https://doi.org/10.1101/458794; this version posted November 7, 2018. The copyright holder for this preprint (which was not certified by peer review) is the author/funder, who has granted bioRxiv a license to display the preprint in perpetuity. It is made available under aCC-BY-NC-ND 4.0 International license.

abundance with wet season peak about 4 times higher than the dry season Static:
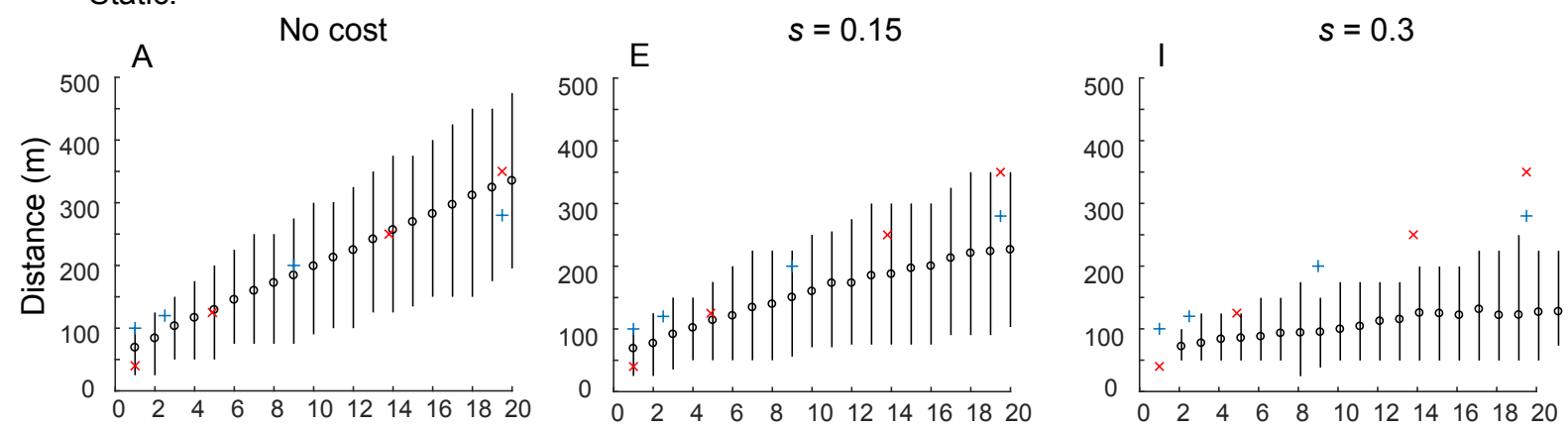

Stochastic (slow):
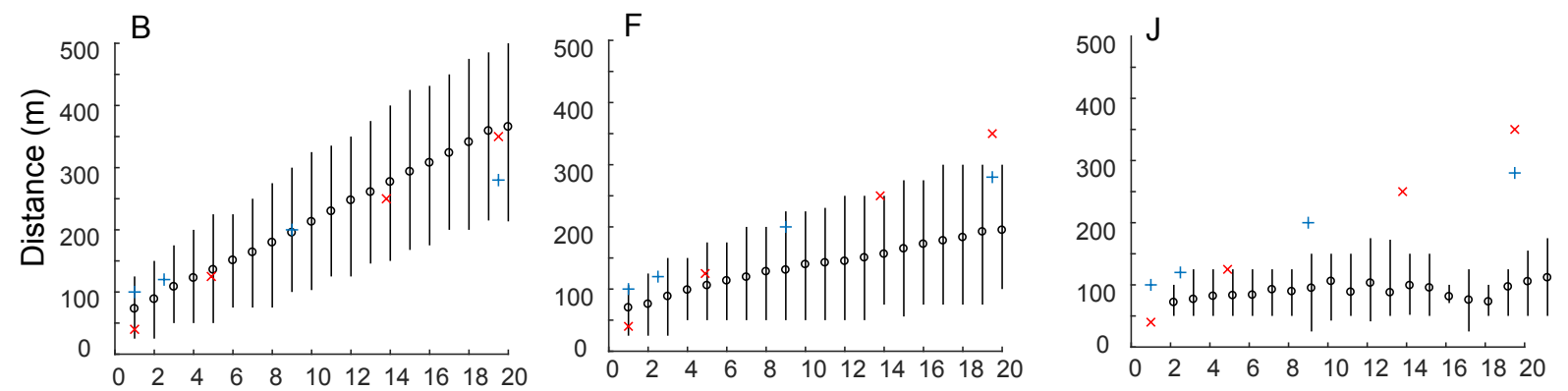

Stochastic (fast):
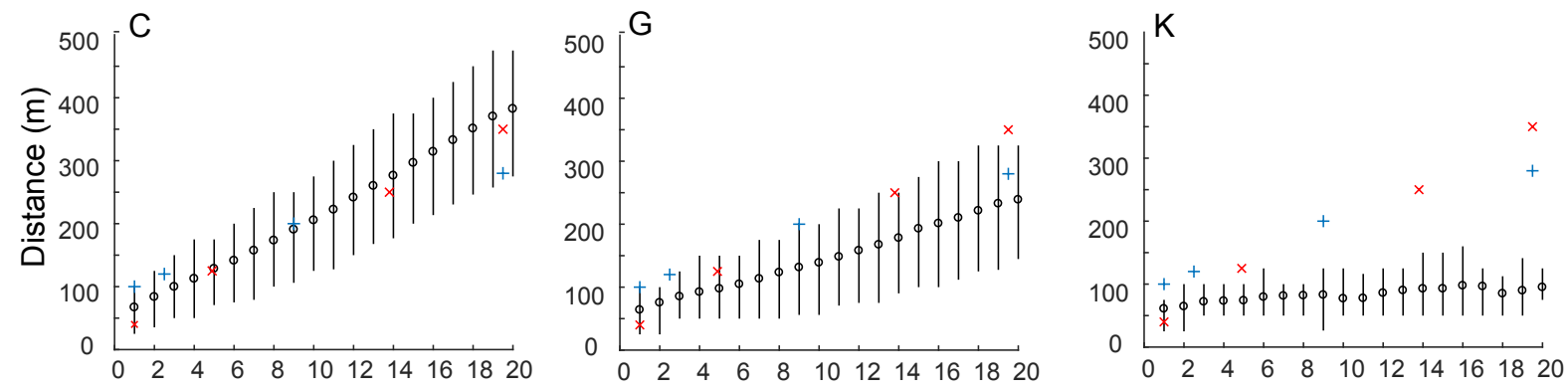

Seasonal:
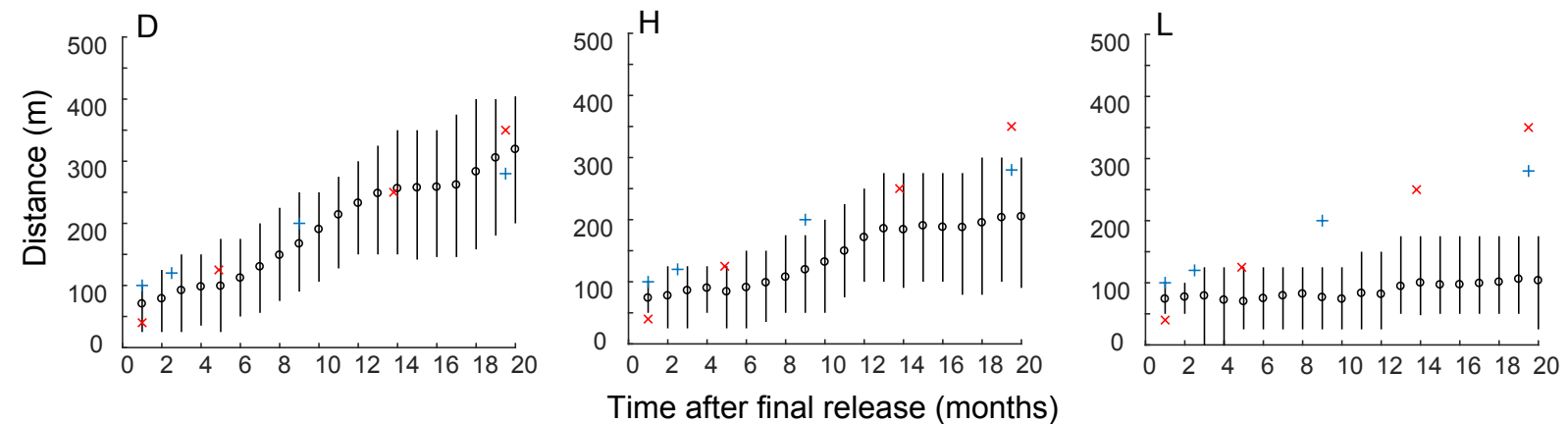

Figure 3. Comparing predicted and observed rates of spatial spread of $w$ Mel Wolbachia. Black circles show the mean across all simulations of the distance of the $0.5 \mathrm{w}$ Mel frequency contour from the edge of the release area and vertical black lines connect the $5 \%$ and $95 \%$ quantiles. The observed mean position of the $0.5 \mathrm{wMel}$ frequency contour from the edge of the release area following the $w$ Mel releases conducted in Cairns is shown for two release areas, Edge Hill (red crosses) and Parramatta Park (blue crosses). Four types of temporal variation in house habitat quality are shown: Static habitat quality (Top row $(\mathbf{A}, \mathbf{E}, \mathbf{I})$ ); Slow stochastic temporal variation ( $\bar{t}_{q}=3$ months; Second row (B,F,J)); Fast stochastic temporal variation $\left(\bar{t}_{q}=1\right.$ month; Second row 
$(\mathbf{C}, \mathbf{G}, \mathbf{K})$ ); Seasonal variation (Fourth row $(\mathbf{D}, \mathbf{H}, \mathbf{L})$ ). Three values of the fitness cost incurred by Wolbachia are shown: No cost ( $s=0$; First column (A-D)); $s=0.15$ (Second column (E-H)); $s=0.3$ (Third column (I-L)).

trough (Fig. S3). Published observations of the seasonal variation in the numbers of Ae aegypti caught in Biogents Sentinal traps (BGS) throughout Cairns show a similar relative amplitude across seasons (Fig. 1 of Ritchie et al. (2013b)). We again simulated the above Wolbachia field release protocol, starting the releases 40 days into the wet season, and again found that the predicted rate of spatial spread of Wolbachia was similar to that obtained when habitat quality was assumed not to vary temporally (Fig. 3D).

\section{Effects of fitness costs}

We then investigated how these spread patterns are affected by fitness costs imposed by Wolbachia on its mosquito host. Specifically, we assumed that Wolbachia-infected individuals experience a reduction in average adult lifespan relative to uninfected individuals by a proportion $s$. Estimates of fitness costs incurred by $w \mathrm{Mel}$ on Ae aegypti are highly uncertain (Hoffmann et al. 2011; Hancock et al. 2016b; Schmidt et al. 2017) (and see the Discussion) but high costs are unlikely; here we consider values of $s=0.15$ and $s=0.3$. For all four types of temporal variation in mosquito habitat quality considered above (including static landscapes with no temporal variation), we simulated the Wolbachia release protocol previously described, but augmented the total release size so that the Wolbachia frequency reached the required value at the time of the final release (Table 2).

For environments where the habitat quality landscape is static, either fitness cost resulted in slower rates of Wolbachia spread that show less agreement with the rates observed in the field populations (Figs. 3E \& 3I). When $s=0.15$ the observed spread rates are above the mean of the simulated rates, but within the $95 \%$ quantiles (Fig. 3E), and when $s=0.3$ the observed rates are higher than the quantiles of the simulated rates for times later than six months following the final release (Fig. 3I).

In the presence of fitness costs, predicted Wolbachia spread rates also differ across the different types of spatio-temporal variation in mosquito habitat quality. They are slower in landscapes where house habitat quality undergoes stochastic changes over time compared to landscapes where house habitat quality is static, with differences being larger when the fitness cost is higher (Figs. 3F-G \& 3J-K). Seasonal variation in mosquito habitat quality also results in slower predicted spread rates, with the difference again being strongest for higher fitness costs (Figs. 3H \& 3L). When the Wolbachia imposes fitness costs, these types of temporal variation in habitat quality result in slower rates of spatial spread because a reduction in a house's habitat quality causes a localized increase in mosquito mortality. If this occurs before the Wolbachia has spread to fixation, the frequency dependent advantage that the Wolbachia has gained by invading and spreading in areas with good habitat quality is lost; thus spread slows down, and may fail if frequencies fall too low. Interestingly, the higher rates of mosquito mortality in temporally variable habitat quality landscapes 
lead to reductions in the intensity of density-dependent larval competition, which can lead to faster Wolbachia spread rates (Hancock et al. 2016b). However in the presence of fitness costs this effect did not compensate for the inhibitory effects on rates of spread of temporal changes in house habitat quality.

\section{DISCUSSION}

Our metapopulation model describes the dynamics of Ae. aegypti populations with spatially heterogeneous abundance and demography, incorporating empirically derived relationships that model density-dependent demographic traits. We demonstrated that the model could approximate the patterns of demographic variation that occur in field populations and predict rates of spatial spread of Wolbachia close to those observed following field releases. We discuss below how our representation of spatially structured population dynamics is important to predicting Wolbachia spread, and also identify limitations of our model in describing the dynamics of natural populations.

Previous studies have demonstrated several mechanisms through which the dynamics of Wolbachia spread are affected by spatial heterogeneity in host abundance (Barton 1979; Hancock \& Godfray 2012), and demographic heterogeneity across individuals (Hancock et al. 2016a; Hancock et al. 2016b). Our modelling approach empirically relates variation in mosquito abundance to density-dependent variation in two demographic traits, namely larval development times and per-capita female fecundity, which are major determinants of the rate of Wolbachia transmission through successive (overlapping) mosquito generations. By allowing density dependence relationships to vary across subpopulations, our model is able to represent realistically two important demographic features of Ae. aegypti field populations: (i) highly variable, spatially aggregated patterns of abundance; and (ii) a relatively intense level of density-dependent larval resource competition on average across the metapopulation.

However, other aspects of demographic and environmental variation that were not included in our model may also cause variable rates of Wolbachia spread. Mosquito mortality and dispersal rates also affect Wolbachia spread dynamics (Schofield 2002; Hancock et al. 2016b), but these traits are poorly quantified and likely to vary with changing environmental conditions (Harrington et al. 2005; Maciel-De-Freitas et al. 2007; Hoffmann 2014). Several demographic traits vary due to changes in weather and climate (Scott et al. 2000a; Mohammed \& Chadee 2011), and predation (Bowatte et al. 2013). Moreover, density dependence may impact other demographic traits such as stage-specific daily survival rates (Hancock et al. 2016a), and mating success (Segoli et al. 2014). Densitydependent dynamics may be further complicated by preferential oviposition behaviour by adult females, whereby females are more likely to oviposit in containers that are already inhabited by conspecific larvae (Wong et al. 2011). Thus our model cannot fully describe the demographic variability in a particular metapopulation. By specifying realistic forms of density dependence, however, our model offers a useful tool for exploring dynamic behaviour across different 
assumptions about uncertain aspects of mosquito-Wolbachia demography and different types of environmental variation.

A current challenge in forecasting Wolbachia dynamics is the difficulty of estimating fitness costs in the field (Hancock et al. 2016b), which is exacerbated by the very high levels of demographic variation caused by environmental and genetic effects (Hoffmann et al. 2011; Walker et al. 2011; Hoffmann 2014; Hancock et al. 2016b; Ross et al. 2016; Schmidt et al. 2017). This is problematic because theory suggests that even small fitness costs strongly affect the spatial spread of Wolbachia (Turelli \& Hoffmann 1991; Hancock \& Godfray 2012). For example, the classical reaction-diffusion models of Barton and Turelli (Barton 1979; Turelli \& Hoffmann 1991) predict that a fitness cost of 0.25 halves the equilibrium wave speed of Wolbachia. These reaction-diffusion approaches assume spatial and temporal constancy of mosquito abundance and demography (Turelli \& Barton 2017). Under plausible sets of demographic and environmental parameters (Tables $1 \& 2$ ), and across a range of models of spatio-temporal habitat variation, our model accurately described observed spread rates without assuming that Wolbachia incurs fitness costs. It is important to note that, while Ae. aegypti abundance varies seasonally in northeast Australia, in many dengueendemic regions (such as parts of Thailand and Peru) abundance does not show a strong seasonal signal (Morrison et al. 2004; Koenraadt et al. 2008). Interestingly, however, for cases where the fitness cost incurred by Wolbachia was low, our predicted spread rates were relatively robust to the different forms of seasonal and stochastic habitat variation that we considered. While our results predict that fitness costs incurred by $w$ Mel are usually small, the uncertainty surrounding the demographic and environmental parameters and processes that we aim to model prevents us from making accurate inferences about the effects of Wolbachia on fitness experienced in field Ae. aegypti populations.

In summary, we present a mathematical model of the spatial dynamics of Wolbachia in Ae. aegypti populations that incorporates realistic features of mosquito demographic variation that impact Wolbachia spread. Our approach facilitates further exploration of the effects of demographic and environmental heterogeneity on the spread of Wolbachia following field releases. Our model can also be applied to explore the dynamics of other mosquito control interventions, such as the release of Wolbachia-infected male mosquitoes in order to suppress vector abundance, or the release of mosquitoes carrying gene drive constructs that aim to suppress abundance or lower vectorial capacity (Flores \& O'Neill 2018). By developing our understanding of the ecological dynamics of Ae. aegypti arbovirus vector populations, and their interactions with vector control interventions, our models can contribute to interpreting observed impacts in field populations and allowing effects of environmental heterogeneity to be considered in designing intervention strategies.

\section{AUTHOR CONTRIBUTIONS}

PAH, HCG and SAR conceived the ideas; PAH designed the methodology; PAH collected the data; PAH analysed the data; PAH led the writing of the manuscript. All authors contributed critically to the drafts and gave final approval for publication. 


\section{ACKNOWLEDGEMENTS}

This research was supported by a Marie Curie International Outgoing Fellowship within the 7th European Community Framework Programme (Grant no. 326551WOLBACHIA-MOD).

\section{DATA ACCESSIBILITY}

Mosquito age-class abundances for LF Population are available on Figshare. $\mathrm{C}++$ Computer Code for implementing the metapopulation model is available on Figshare.

\section{REFERENCES}

Aldstadt, J., Koenraadt, C.J.M., Fansiri, T., Kijchalao, U., Richardson, J., Jones, J.W. \& Scott, T.W. (2011) Ecological Modeling of Aedes aegypti (L.) Pupal Production in Rural Kamphaeng Phet, Thailand. PLoS Negl Trop Dis, 5.

Arrivillaga, J. \& Barrera, R. (2004) Food as a limiting factor for Aedes aegypti in water-storage containers. J Vector Ecol, 29, 11-20.

Barrera, R., Amador, M. \& Clark, G.G. (2006) Ecological factors influencing Aedes aegypti (Diptera : Culicidae) productivity in artificial containers in Salinas, Puerto Rico.J Med Entomol, 43, 484-492.

Barton, N.H. (1979) The dynamics of hybrid zones. Heredity, 43, 341-359.

Bhatt, S., Gething, P.W., Brady, O.J., Messina, J.P., Farlow, A.W., Moyes, C.L., . . Hay, S.I. (2013) The global distribution and burden of dengue. Nature, 496, 504-507.

Bowatte, G., Perera, P., Senevirathne, G., Meegaskumbura, S. \& Meegaskumbura, M. (2013) Tadpoles as dengue mosquito (Aedes aegypti) egg predators. Biological Control, 67, 469-474.

Briegel, H. (1990) Metabolic relationship between female body size, reserves, and fecundity of Aedes aegypti. J Insect Physiol, 36, 165-172.

Caspari, E. \& Watson, G.S. (1959) On the evolutionary importance of cytoplasmic sterility in mosquitoes. Evolution, 13, 568-570.

Darbro, J.M., Johnson, P.H., Thomas, M.B., Ritchie, S.A., Kay, B.H. \& Ryan, P.A. (2012) Effects of Beauveria bassiana on Survival, Blood-Feeding Success, and Fecundity of Aedes aegypti in Laboratory and Semi-Field Conditions. Am J Trop Med Hyg, 86, 656-664.

Faria, N.R., Quick, J., Claro, I.M., Theze, J., de Jesus, J.G., Giovanetti, M., . . Pybus, O.G. (2017) Establishment and cryptic transmission of Zika virus in Brazil and the Americas. Nature, 546, 406-+.

Flores, H.A. \& O'Neill, S.L. (2018) Controlling vector-borne diseases by releasing modified mosquitoes. Nature Reviews Microbiology, 16, 508-518.

Focks, D.A. \& Alexander, N. (2006) Multicountry study of Aedes aegypti pupal productivity: findings and recommendations. World Health Organization.

Getis, A., Morrison, A.C., Gray, K. \& Scott, T.W. (2003) Characteristics of the spatial pattern of the dengue vector, Aedes aegypti, in Iquitos, Peru. Am J Trop Med Hyg, 69, 494-505.

Hancock, P.A. \& Godfray, H.C.J. (2012) Modelling the spread of Wolbachia in spatially heterogeneous environments. J Royal Soc Interface, 9, 30453054. 
Hancock, P.A., Linley-White, V., Callahan, A.G., Godfray, H.C.J., Hoffmann, A.A. \& Ritchie, S.A. (2016a) Density-dependent population dynamics in Aedes aegypti slow the spread of $w$ Mel Wolbachia. J Appl Ecol, 53, 785-793.

Hancock, P.A., White, V.L., Ritchie, S.A., Hoffmann, A.A. \& Godfray, H.C.J. (2016b) Predicting Wolbachia invasion dynamics in Aedes aegypti populations using models of density-dependent demographic traits. BMC Biology, 14.

Harrington, L.C., Scott, T.W., Lerdthusnee, K., Coleman, R.C., Costero, A., Clark, G.G., ... Edman, J.D. (2005) Dispersal of the dengue vector Aedes aegypti within and between rural communities. Am J Trop Med Hyg, 72, 209-220.

Hoffmann, A.A. (2014) Facilitating Wolbachia invasions. Austral Entomology, 53, 125-132.

Hoffmann, A.A., Montgomery, B.L., Popovici, J., Iturbe-Ormaetxe, I., Johnson, P.H., Muzzi, F., ... O'Neill, S.L. (2011) Successful establishment of Wolbachia in Aedes populations to suppress dengue transmission. Nature, 476, 454U107.

Jeffery, J.A.L., Nguyen Thi, Y., Vu Sinh, N., Le Trung, N., Hoffmann, A.A., Kay, B.H. \& Ryan, P.A. (2009) Characterizing the Aedes aegypti population in a Vietnamese village in preparation for a Wolbachia-based mosquito control strategy to eliminate dengue. PLoS Negl Trop Dis, 3, e552.

Koenraadt, C.J.M., Aldstadt, J., Kijchalao, U., Sithiprasasna, R., Getis, A., Jones, J.W. \& Scott, T.W. (2008) Spatial and temporal patterns in pupal and adult production of the dengue vector Aedes aegypti in Kamphaeng Phet, Thailand. Am J Trop Med Hyg, 79, 230-238.

LaCon, G., Morrison, A.C., Astete, H., Stoddard, S.T., Paz-Soldan, V.A., Elder, J.P., . . Vazquez-Prokopec, G.M. (2014) Shifting Patterns of Aedes aegypti Fine Scale Spatial Clustering in Iquitos, Peru. PLoS Negl Trop Dis, 8.

Legros, M., Lloyd, A.L., Huang, Y.X. \& Gould, F. (2009) Density-dependent intraspecific competition in the larval stage of Aedes aegypti (Diptera: Culicidae): Revisiting the current paradigm. J Med Entomol, 46, 409-419.

Maciel-De-Freitas, R., Codeco, C.T. \& Lourenco-De-Oliveira, R. (2007) Daily survival rates and dispersal of Aedes aegypti females in Rio de Janeiro, Brazil. Am J Trop Med Hyg, 76, 659-665.

Magori, K., Legros, M., Puente, M.E., Focks, D.A., Scott, T.W., Lloyd, A.L. \& Gould, F. (2009) Skeeter Buster: A Stochastic, Spatially Explicit Modeling Tool for Studying Aedes aegypti Population Replacement and Population Suppression Strategies. PLoS Negl Trop Dis, 3, Article No.: e508.

Mohammed, A. \& Chadee, D.D. (2011) Effects of different temperature regimens on the development of Aedes aegypti (L.) (Diptera: Culicidae) mosquitoes. Acta Trop, 119, 38-43.

Moreira, L.A., Iturbe-Ormaetxe, I., Jeffery, J.A., Lu, G.J., Pyke, A.T., Hedges, L.M., ... O'Neill, S.L. (2009) A Wolbachia symbiont in Aedes aegypti limits infection with Dengue, Chikungunya, and Plasmodium. Cell, 139, 1268-1278.

Morrison, A.C., Gray, K., Getis, A., Astete, H., Sihuincha, M., Focks, D., . . Scott, T.V. (2004) Temporal and geographic patterns of Aedes aegypti (Diptera : Culicidae) production in Iquitos, Peru. J Med Ent, 41, 1123-1142.

Muir, L.E. \& Kay, B.H. (1998) Aedes aegypti survival and dispersal estimated by mark-release-recapture in northern Australia. Am J Trop Med Hyg, 58, 277-282. 
Muriu, S.M., Coulson, T., Mbogo, C.M. \& Godfray, H.C.J. (2013) Larval density dependence in Anopheles gambiae s.s., the major African vector of malaria. J Anim Ecol, 82, 166-174.

Nasci, R.S. (1986) The size of emerging and host-seeking Aedes-aegypti and the relation of size to blood-feeding success in the field. J Am Mosq Control Assoc, 2, 61-62.

Nunes, M.R.T., Faria, N.R., de Vasconcelos, J.M., Golding, N., Kraemer, M.U.G., de Oliveira, L.F., ... Vasconcelos, P.F.D. (2015) Emergence and potential for spread of Chikungunya virus in Brazil. BMC Med, 13.

O"Neill, S.L., Ryan, P.A., Turley, A.P., Wilson, G., Retski, K., Iturbe-Ormaetxe, I., . . . Simmons, C.P. (2018) Scaled deployment of Wolbachia to protect the community from Aedes transmitted arboviruses. Gates Open Res, 2:36.

Ritchie, S.A., Montgomery, B.L. \& Hoffmann, A.A. (2013a) Novel Estimates of Aedes aegypti (Diptera: Culicidae) Population Size and Adult Survival Based on Wolbachia Releases. J Med Ent, 50, 624-631.

Ritchie, S.A., Pyke, A.T., Hall-Mendelin, S., Day, A., Mores, C.N., Christofferson, R.C., ... van den Hurk, A.F. (2013b) An Explosive Epidemic of DENV-3 in Cairns, Australia. Plos One, 8.

Ross, P.A., Endersby, N.M. \& Hoffmann, A.A. (2016) Costs of Three Wolbachia Infections on the Survival of Aedes aegypti Larvae under Starvation Conditions. PLoS Negl Trop Dis, 10.

Saez-Llorens, X., Tricou, V., Yu, D., Rivera, L., Tuboi, S., Garbes, P., ... Wallace, D. (2017) Safety and immunogenicity of one versus two doses of Takeda's tetravalent dengue vaccine in children in Asia and Latin America: interim results from a phase 2, randomised, placebo-controlled study. Lancet Infect Dis, 17, 615-625.

Schmidt, T.L., Barton, N.H., Rasic, G., Turley, A.P., Montgomery, B.L., IturbeOrmaetxe, I., ... Turelli, M. (2017) Local introduction and heterogeneous spatial spread of dengue-suppressing Wolbachia through an urban population of Aedes aegypti. PLoS Biol, 15.

Schneider, J.R., Morrison, A.C., Astete, H., Scott, T.W. \& Wilson, M.L. (2004) Adult size and distribution of Aedes aegypti (Diptera : Culicidae) associated with larval habitats in Iquitos, Peru. J Med Ent, 41, 634-642.

Schofield, P. (2002) Spatially explicit models of Turelli-Hoffmann Wolbachia invasive wave fronts. J Theor Biol, 215, 121-131.

Scott, T.W., Amerasinghe, P.H., Morrison, A.C., Lorenz, L.H., Clark, G.G., Strickman, D., ... Edman, J.D. (2000a) Longitudinal studies of Aedes aegypti (Diptera : Culicidae) in Thailand and Puerto Rico: Blood feeding frequency.J Med Ent, 37, 89-101.

Scott, T.W., Morrison, A.C., Lorenz, L.H., Clark, G.G., Strickman, D., Kittayapong, P., ... Edman, J.D. (2000b) Longitudinal studies of Aedes aegypti (Diptera : Culicidae) in Thailand and Puerto Rico: Population dynamics. J Med Ent, 37, 77-88.

Segoli, M., Hoffmann, A.A., Lloyd, J., Omodei, G.J. \& Ritchie, S.A. (2014) The Effect of Virus-Blocking Wolbachia on Male Competitiveness of the Dengue Vector Mosquito, Aedes aegypti. PLoS Negl Trop Dis, 8, E3294-E3294.

Southwood, T.R.E., Murdie, G., Yasuno, M., Tonn, R.J. \& Reader, P.M. (1972) Studies on the life budget of Aedes aegypti in Wat Samphaya, Bangkok, Thailand. Bull World Health Org, 46, 211-226. 
Turelli, M. (1994) Evolution of incompatibility inducing microbes and their hosts. Evolution, 48, 1500-1513.

Turelli, M. \& Barton, N.H. (2017) Deploying dengue-suppressing Wolbachia: Robust models predict slow but effective spatial spread in Aedes aegypti. Theor Popul Biol, 115, 45-60.

Turelli, M. \& Hoffmann, A.A. (1991) Rapid spread of an inherited incompatability factor in California Drosophila. Nature, 353, 440-442.

Walker, T., Johnson, P.H., Moreira, L.A., Iturbe-Ormaetxe, I., Frentiu, F.D., McMeniman, C.J., ... Hoffmann, A.A. (2011) The wMel Wolbachia strain blocks dengue and invades caged Aedes aegypti populations. Nature, 476, 450-U101.

Williams, C.R., Johnson, P.H., Ball, T.S. \& Ritchie, S.A. (2013) Productivity and population density estimates of the dengue vector mosquito Aedes aegypti (Stegomyia aegypti) in Australia. Med Vet Ent, 27, 313-322.

Wong, J., Stoddard, S.T., Astete, H., Morrison, A.C. \& Scott, T.W. (2011) Oviposition Site Selection by the Dengue Vector Aedes aegypti and Its Implications for Dengue Control. PLoS Negl Trop Dis, 5. 